SEP 041990

\title{
IS THERE EVIDENCE FOR FLOW IN HEAVY ION COLLISIONS?
}

\author{
H. Von Gersdorff \\ Physics Department \\ Brookhaven National Laboratory \\ Upton, New York 11973
}

\begin{abstract}
Calculations using a transverse hydrodynamical model with a phase transition in the equation of state are compared to recent heavy ion data. We argue that hydrodynamics alone cannot reproduce the low $p_{T}$ part of the experimental spectrum.
\end{abstract}

Invited talk presented at

Quark Matter 90

Menton, France, 7-11 May i990

This manuscript has been authored under contract number DE-AC02-76CH00016 with the U.S. Department of Energy. Accordingly, the U.S. Government retains a non-exclusive, royalty-free license to publish or reproduce the published form of this contribution, or allow others to do so, for U.S. Government purposes. 


\section{IS THERE EVIDENCE FOR FLOW IN HEAVY ION COLLISIONS?}

\section{Henrique VON GERSDORFF}

Brookhaven National Laboratory, Physics Department, Upton, N.Y., 11973

Calculations using a transverse hydrodynamical model with a phase transition in the equation of state are compared to recent heavy ion data. We argue that hydrodynamics alone cannot reproduce the low $p_{T}$ part of the experimental spectrum.

Clear evidence for the collective phenomenon of flow in heavy ion collisions has been found ${ }^{1}$ for ${ }^{93} \mathrm{Nb}+{ }^{93} \mathrm{Nb}$ central collisions at $400 \mathrm{~A} \mathrm{MeV}$. It was also found that ${ }^{40} \mathrm{Ca}+{ }^{40} \mathrm{Ca}$ central collisions exhibit a much weaker flow effect at the same bombardment energy, so that large nuclei, which endure longer as a cohesive whole before the final decoupling into the observed secondaries, were crucial in the conclusive establishment of flow in these reactions. One concludes that to get collective flow one needs a copious amount of rescatterings among the constituent particles of the fireball formed by the collision.

At higher energies pion production dominates the multiplicity and the previous analysis of flow becomes untenable. Nevertheless, the highly excited fireball still has to expand and cool off before it breaks up, since if it breaks up immediately after the collision its temperature and therefore the average transverse momentum $\left\langle p_{T}\right\rangle$ of the secondaries will be too high to agree with experiment. Moreover, as emphasized by Pomeranchuk, too many heavy particles would be produced without a preliminary expansion. A collective flow should therefore also develop at higher energies in the initial stages of the collision when the constituents of the system are still interacting strongly. The search for evidence of this expected behavior is the main motivation behind this paper and a of series of others.

The observable we will calculate in this search for evidence of flow is the $p_{T}$ distribution of the produced secondaries. The data is from the NA35 collaboration ${ }^{4}$ and was taken over the central rapidity range $2 \leq y \leq 3$, where $\mathrm{d} N / \mathrm{d} y$ is approximately constant as we assume in our longitudinal boost invariant hydrodynamics.

First suppose a hot and non-interacting pion gas is created by the collision and its subsequent expansion is governed by hydrodynamics. Initially, the pressure $\left(P \sim T^{4}\right)$ will be very high and thus the expansion very fast. At a later stage, after the gas has cooled, the expansion will be quite weak, until eventually the mean free path of the pions becomes comparable to that of the system which then decouples into the free streaming pions observed by the detectors. To estimate the initial energy density one uses the experimentally known

This manuscript has been authored under contract number DE-AC02-76CH00016 with the U.S. Department of Energy. 
total multiplicity per unit rapidity $\mathrm{d} N / \mathrm{d} y$ and entropy conservation. Since $\mathrm{d} S / \mathrm{d} y \simeq 3.6$ $\mathrm{d} N / \mathrm{d} y$ (massless boson gas) and $\mathrm{d} S / \mathrm{d} y=\pi R^{2} \tau_{0} s_{0}$ (where $\mathrm{R}$ is the nuclear radius, $\tau_{0}$ the initial formation proper time and $s_{0}$ the corresponding initial entropy density) one finds that

$$
s_{0} \simeq \frac{3.6}{\pi R^{2} \tau_{0}} \frac{\mathrm{d} N}{\mathrm{~d} y}
$$

The NA35 data for ${ }^{32} \mathrm{~S}+{ }^{32} \mathrm{~S}$ collisions at $200 \mathrm{~A} \mathrm{GeV}$ has a $\mathrm{d} N / \mathrm{d} y \simeq 30$ at mid-rapidity ( $y=3.03$ ) for negatively charged particles ( $90 \%$ pions), so that the total $\mathrm{d} N / \mathrm{d} y \simeq 90$. Assuming $\tau_{0}=1.25 \mathrm{fm} / \mathrm{c}$ and $R=3.5 \mathrm{fm}$, one finds from Eq. (1) that $s_{0}=6.7 \mathrm{fm}^{-3}=$ $g_{\pi}\left(4 \pi^{2} / 90\right) T^{3}$ which gives $T \simeq 334 \mathrm{MeV}\left(g_{\pi}=3\right)$ and initial energy density $\epsilon_{0}=\frac{3}{4} s_{0} T \simeq 1.6$ $\mathrm{GeV} / \mathrm{fm}^{3}$

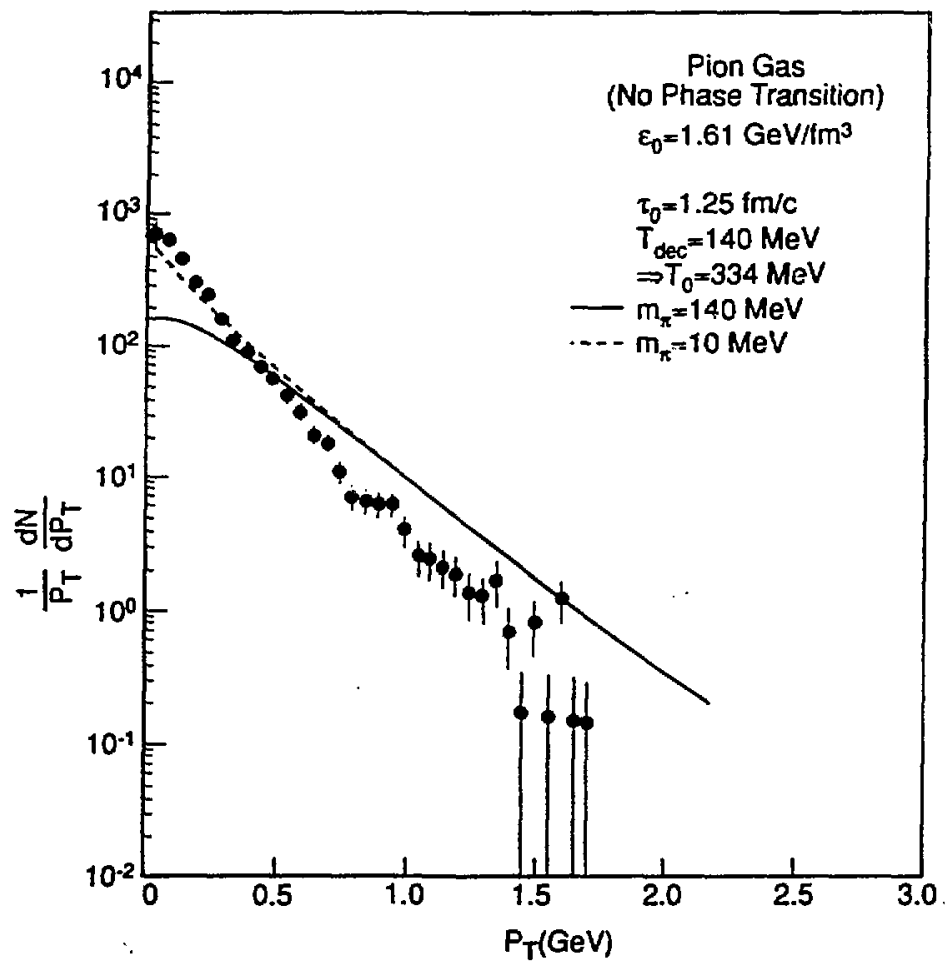

Figure 1: The $p_{T}$ distribution for a pion gas and the NA35 $\mathrm{S}+\mathrm{S}$ preliminary data for negatively charged particles at $200 \mathrm{~A} \mathrm{GeV}$.

This temperature is barely consistent with a super-heated pion gas and for values larger than this the system cannot be considered an ideal gas since interactions cannot be neglected when the density of the gas is comparable to that of a pion (extra hadronic resonances like $\rho, \omega$ and $\eta$ must be included, thereby reducing the initial temperature of the gas). Nevertheless, we plot in Fig. 1 the $p_{T}$ distribution calculated with the above initial conditions 
supplemented with a decoupling temperature $T_{\text {dec }}=140 \mathrm{MeV}$ and compare it to the preliminary NA34 ${ }^{32} \mathrm{~S}+{ }^{32} \mathrm{~S}$ data. Notice the slight positive curvature of the distribution in the range $0.5 \mathrm{GeV} / \mathrm{c}<p_{T}<2.0 \mathrm{GeV} / \approx$ and the large difference between the massive and massless cases. At high $p_{T}$ the pion mass is comparatively small so that the two scales coincide. Integrating the curve for the massless case $\mathrm{d} N / \mathrm{d} y=91$ and the $\left\langle p_{T}\right\rangle=455 \mathrm{MeV} / \mathrm{c}$, so that the pion gas assumption underestimates the contribution of the low $p_{T}$ component of the distribution and overestimates the high $p_{T}$ component.

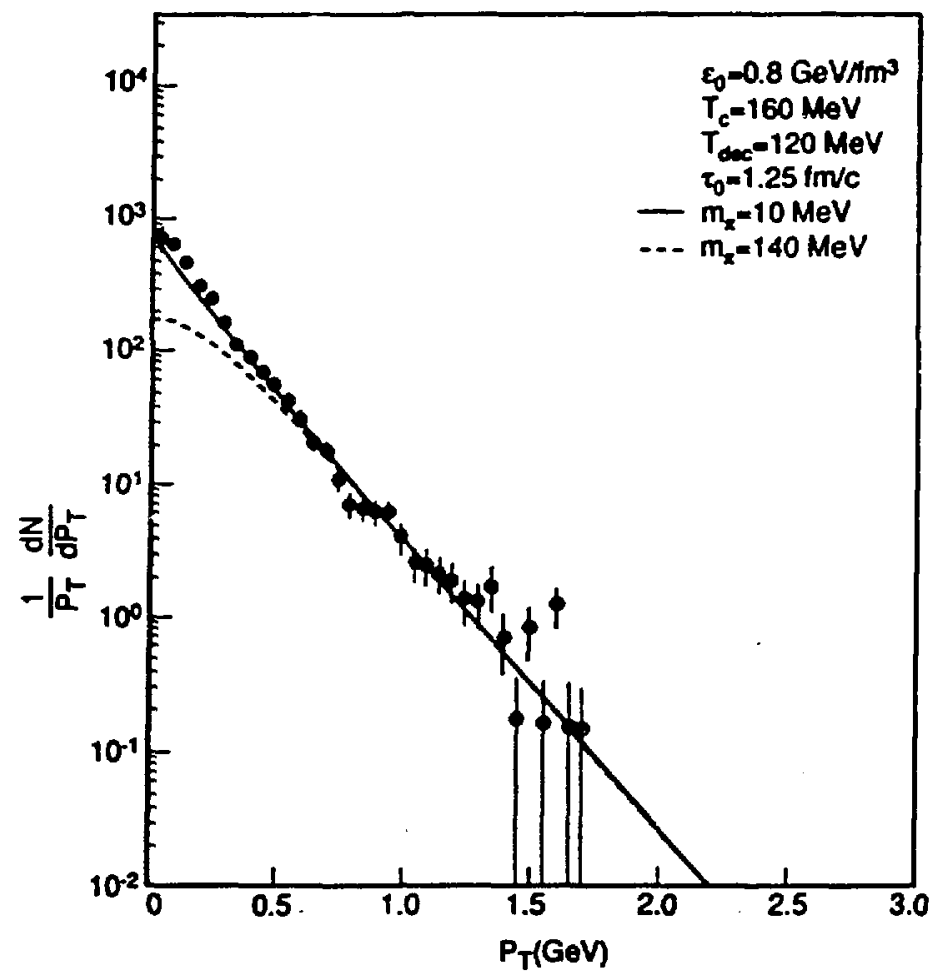

Figure 2: The $p_{T}$ distribution for a mixed phase scenario.

If, instead of a pure pion gas, a first order phase transition to a quark-gluon plasma occurs at $T_{c}=160 \mathrm{MeV}$, the critical entropy density for the pion and quark gluon phases are: $s_{\pi}\left(T_{c}\right)=0.704 \mathrm{fm}^{-3}$ and $s_{q g}\left(T_{c}\right)=8.6 \mathrm{fm}^{-3}$ The initial high density state formed by the ${ }^{32} \mathrm{~S}+{ }^{32} \mathrm{~S}$ collisions at $200 \mathrm{~A} \mathrm{GeV}$ is thus a mixed phase of pions and quark-gluon plasma. From the equation $6.7=0.704(1-f)+8.6 f$, we obtain the fraction of the matter that is in the quark-gluon plasma phase, $f=76 \%$. The initial energy density can now be calculated: $\epsilon_{0}=\epsilon_{\pi}(1-f)+\epsilon_{g g} f$, where $\epsilon_{\pi}=0.084 \mathrm{GeV} / \mathrm{fm}^{3}$ and $\epsilon_{q g}=1.03 \mathrm{GeV} / \mathrm{fm}^{3}$, which results in $\epsilon_{0}=0.8 \mathrm{GeV} / \mathrm{fm}^{3}$. In Fig. 2 we show the $p_{T}$ distribution one finds assuming these initial conditions and a decoupling temperature of $T_{\text {dec }}=12 \mathrm{~J} \mathrm{MeV}$. For the massless case $\left(m_{\pi}=10 \mathrm{MeV}\right.$ to avoid divergences in the decoupling integral) $\mathrm{d} N / \mathrm{d} y=75$ and $\left\langle p_{T}\right\rangle=330$ 
$\mathrm{Mev} / \mathrm{c}$, whereas the experimental values are total $\mathrm{d} N / \mathrm{d} y=3 \times 28=84$ and $\left\langle p_{T}\right\rangle=350$ $\mathrm{MeV}$. When the pion mass is included a disagreement of almost an order of magnitude with the data is found at low $\left\langle p_{T}\right\rangle$, where the bulk of the multiplicity resides. The enhancement of the low $p_{T}$ component for the massless case is due to the singular behavior of the BoseEinstein distribution in the decoupling integral in the limit when $p_{T}$ and $m_{\pi}$ vanish. The striking feature of this curve is its complete lack of curvature, except at very low $p_{T}$ values. Compared to the pure pion gas, a much softer flow is now generated. This is due to the mixed phase which causes the system to last longer (the velocity of sound vanishes), thus making it more susceptible to the $p_{T}$ attenuating effects of the longitudinal expansion. A suppression of strong transverse flow effects is thus obtained.

In conclusion we emphasize the following points: (i) A non-zero collective flow velocity tends to give transverse momentum distributions a concave shape for effectively massless particles. (ii) An ideal pion gas is ruled out by the data since it generates too strong a transverse flow. (iii) The presence of a mixed phase in the equation of state tends to soften the transverse flow. On a semi-log plot the distributions have a linear behavior for a large $p_{T}$ range and are thus nearly indistinguishable from the thermal model ones, which have zero flow velocity6 (iv) The non-zero slope of the experimental $p_{T}$ distribution at small $p_{T}$ values cannot be understand solely within the framework of relativistic hydrodynamics. Some new physics is needed here (note that we have ignored entirely the role of nucleons and of interactions among the constituent particles). (v) No unambiguous evidence for collective flow has emerged from our analysis. Perhaps this will only happen when higher energies and heavier nuclei become available at CERN and RHIC.

\section{REFERENCES}

1. H. A. Gustafson et al., Phys. Rev. Lett. $\underline{52}$ (1984) 1590.

2. I. Ya. Pomeranchuk, Dokl. Akad. Nauk SSSR $\underline{78}$ (1951) 889.

3. E. F. Staubo et al., Phys. Lett. B229 (1989) 351;

K. S. Lee, U. Heinz and E. Schnedermann, Regensburg preprint (1990)

to appear in Z. Phys. C;

R. Venugopalan and M. Prakash, Phys. Rev. C41 (1990) 221;

U. Ornik, F. Pottag and F. Weiner, Phys. Rev. Lett. 63 (1989) 2641;

D. Kusnezov and G. Bertsch, Phys. Rev. C40, (1989) 2075.

4. J. W. Harris (NA35 collab.), Nucl. Phys. A498 (1989) 133 c.

5. P. V. Ruuskanen, Z. Phys. C38 (1988) 219.

6. J.-P. Blaizot and J.-Y. Ollitrault, Nucl. Phys. A458 (1986) 745;

P. V. Ruuskanen, Acía Phy. Polon. B18 (1987) 551.

\section{DISCLAIMER}

This report was prepared as an account of work sponsored by an agency of the United States Government. Neither the United States Government nor any agency thereof, nor any of their employees, makes any warranty, express or implied, or assumes any legal liability or responsibility for the accuracy, compieteness, or usefulness of any information, apparatus, product, or process disclosed, or represents that its use would not infringe privately owned rights. Reference herein to any specific commercial product, process, or service by trade name, trademark, manufacturer, or otherwise does not necessarily constitute or imply its endorsement, recommendation, or favoring by the United States Government or any agency thereof. The views and opinions of authors expressed herein do not necessarily state or reflect those of the United States Government or any agency thereof. 